\title{
Major adverse cardiovascular
} and limb events in patients with diabetes and concomitant peripheral artery disease treated with sodium glucose cotransporter 2 inhibitor versus dipeptidyl peptidase-4 inhibitor

Hsin-Fu Lee 1,2,3,4, Shao-Wei Chen ${ }^{5}$, Jia-Rou Liu' ${ }^{6}$, Pei-Ru Li6 ${ }^{6}$ Lung-Sheng Wu 1,2, Shang-Hung Chang1,2,7, Yung-Hsin Yeh ${ }^{1,2}$, Chi-Tai Kuo ${ }^{1,2}$, Yi-Hsin Chan $^{1,2,8^{*}}$ and Lai-Chu See ${ }^{6,9,10^{*}}$

\begin{abstract}
Background: Whether sodium glucose co-transporter 2 inhibitors (SGLT2i) are associated with a lower risk of cardiovascular as well as adverse lower limb events in patients with type-2 diabetes mellitus (T2DM) and concomitant peripheral artery disease (PAD) is unclear.

We aimed to evaluate the risk of cardiovascular and limb events, and death associated with the use of SGLT2i compared with dipeptidyl peptidase-4 inhibitors (DPP4i) among a longitudinal and national cohort of patients with T2DM.

Methods: In this nationwide retrospective cohort study based on the Taiwan National Health Insurance Research Database, we identified a total of 11,431 and 93,972 consecutive T2DM patients with PAD taking SGLT2i and DPP4i, respectively, from May 1, 2016, to December 31,2017. We used 1:1 propensity score matching (PSM) to balance covariates across study groups. Patients were followed from the drug index date until the occurrence of clinical outcomes, death, discontinuation of the index drug, or the end of the study period, whichever occurred first.

Results: Overall, $56 \%$ and $44 \%$ of the patients were treated with dapagliflozin and empagliflozin, respectively. The use of SGLT2i had comparable risks of ischemic stroke and acute myocardial infarction, and was associated with lower risks of congestive heart failure (CHF) [hazard ratio (HR): 0.66; 95\% confidence interval (Cl) 0.49-0.89; $p=0.0062$ ], lower limb ischemia requiring revascularization (HR: $0.73 ; 95 \% \mathrm{Cl} 0.54-0.98 ; p=0.0367$ ) or amputation (HR: $0.43 ; 95 \%$ $\mathrm{Cl} 0.30-0.62 ; p<0.0001)$, and cardiovascular death (HR: $0.67 ; 95 \% \mathrm{Cl} 0.49-0.90 ; p=0.0089$ ) when compared with the DDP4i group after PSM. The subgroup analysis revealed consistent results for CHF and major adverse limb outcomes for SGLT2i versus DPP4i among patients aged $\geq 75$ years, the presence of chronic kidney disease and established cardiovascular disease was consistent with the main analysis.
\end{abstract}

\footnotetext{
*Correspondence: s851047@hotmail.com; lichu@mail.cgu.edu.tw

1 The Cardiovascular Department, Chang Gung Memorial Hospital, Linkou, Taoyuan 33305, Taiwan

${ }^{6}$ Department of Public Health, College of Medicine, Chang Gung University, No. 259, Wenhua 1st Rd., Guishan Dist, Taoyuan 33302, Taiwan

Full list of author information is available at the end of the article
}

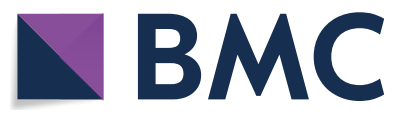

(c) The Author(s) 2020. This article is licensed under a Creative Commons Attribution 4.0 International License, which permits use, sharing, adaptation, distribution and reproduction in any medium or format, as long as you give appropriate credit to the original author(s) and the source, provide a link to the Creative Commons licence, and indicate if changes were made. The images or other third party material in this article are included in the article's Creative Commons licence, unless indicated otherwise in a credit line to the material. If material is not included in the article's Creative Commons licence and your intended use is not permitted by statutory regulation or exceeds the permitted use, you will need to obtain permission directly from the copyright holder. To view a copy of this licence, visit http://creativecommons.org/licenses/by/4.0/. The Creative Commons Public Domain Dedication waiver (http://creativecommons.org/publicdomain/zero/1.0/) applies to the data made available in this article, unless otherwise stated in a credit line to the data. 
Conclusions: SGLT2i were associated with lower risks of CHF and adverse lower limb events compared with DPP4i among patients with T2DM and PAD in real-world practice.

\section{Background}

Sodium-glucose co-transporter-2 inhibitors (SGLT2i) have shown benefits for different endpoints, such as renal outcomes, heart failure, and major cardiovascular $(\mathrm{CV})$ events among patients with type-2 diabetes mellitus (T2DM) treated with antihyperglycemic agents [1-4]. Although the two available trials with dapagliflozin and empagliflozin did not report a significant increase in amputations, the Canagliflozin Cardiovascular Assessment Study (CANVAS) program indicated a higher rate of amputations in the canagliflozin group compared with the placebo group (6.3 vs. 3.4 patients per 1000 patient-years) [1-3]. Nevertheless, the CANVAS results raised concerns regarding the suitability of SGLT2i for patients with T2DM with a high risk of amputation, such as those with concomitant peripheral artery disease (PAD). A few observational studies have investigated the association of SGLT2i with the risk of lower limb amputation; however, these studies have reported inconsistent and conflicting findings. For example, Yuan et al. reported no increased risk of amputations (hazard ratio [HR] 0.98; 95\% confidence interval [CI] 0.68-1.41); Adimadhyam et al. reported increased risk (HR 1.38, 95\% CI 0.83-2.31); and Udell et al. reported an increased risk (HR 1.99, 95\% CI 1.123.51) for SGLT2i treatment compared with nonSGLT2i agents [5-7].

T2DM is a major risk factor for CV disease and PAD, and the prevalence of PAD in patients with T2DM has been estimated to reach $20 \%$ [8-10]. Patients with T2DM and concomitant PAD have an increased risk of $\mathrm{CV}$ events and amputation compared with those without PAD [11]. Subgroup analyses of the landmark studies on empagliflozin revealed consistent $\mathrm{CV}$ benefits in patients with T2DM and concomitant PAD without an increased risk of amputation [12]. However, real-world data on the effectiveness, safety, and limb outcomes for such a specific population treated with SGLT2i are scarce. Dipeptidyl peptidase-4 inhibitors (DPP4i) improve glycemic control by increasing the serum levels of glucagon-like peptide 1 (GLP-1) and exhibit a neural effect in $\mathrm{CV}$ composite outcomes, that are clinically widely prescribed as second-line agents in the management of hyperglycemia for patients with T2DM [13, 14]. Our study investigated the outcomes of patients with T2DM and concomitant PAD treated with SGLT2i compared with those treated with DPP4i in a large, real-world setting.

\section{Methods \\ Study population}

This retrospective nationwide cohort study analyzed data from the Taiwan National Health Insurance (NHI) Research Database (NHIRD), which contains detailed health-care information for more than 23 million enrollees with a $>99 \%$ coverage rate of residents of Taiwan [15]. This study was approved by the Institutional Review Board of Chang Gung Medical Foundation, Taiwan (1048079B and 201801427B0). Informed consent was waived because the original identification number of each patient in the NHIRD had been encrypted and de-identified to protect their privacy.

\section{Study cohort}

The study identified a total of 3,623,527 patients with T2DM diagnosed using International Classification of Diseases (ninth revision) Clinical Modification (ICD9-CM) codes (250) between January 1, 1998 and December 31, 2015, or ICD-10-CM codes (E10.0, E10.1, E10.9, E11.0, E11.1, and E11.9) between January 1, 2016 and December 31, 2017. To identify patients with T2DM who had diagnoses indicating PAD, patients with PAD were required to fulfill with at least one of the following the diagnoses or treatments, which have been registered using medical records, ICD-9-CM or ICD-10-CM diagnostic codes, or ICD-9/10-CM procedural codes (Additional file 1: Table S1). Among the 452,149 patients with T2DM and concomitant PAD, 12,355 patients received first prescriptions of SGLT2i (empagliflozin and dapagliflozin; approval date in Taiwan: May 1, 2016) between May 1, 2016 and December 31, 2017. Canagliflozin has not been included in the present study because it is approved after March 1, 2018 in Taiwan. Of the other 439,794 patients not receiving SGLT2i treatments, 93,972 patients received first prescriptions for DPP4i (saxagliptin, sitagliptin, linagliptin, or alogliptin) during the same period. Patients with T2DM are not allowed to use SGLT2i and DPP4i simultaneously according to Taiwan's NHI regulations. For each study group, the index date was defined as the first date of prescription for SGLT2i or DPP4i after May 1, 2016. The follow-up period was from the index date until the independent occurrence of any study outcome, discontinuation of the index drug, or end date of the study period (December 31, 2017), whichever occurred first. The flowchart of study enrollment is summarized in Fig. 1. 


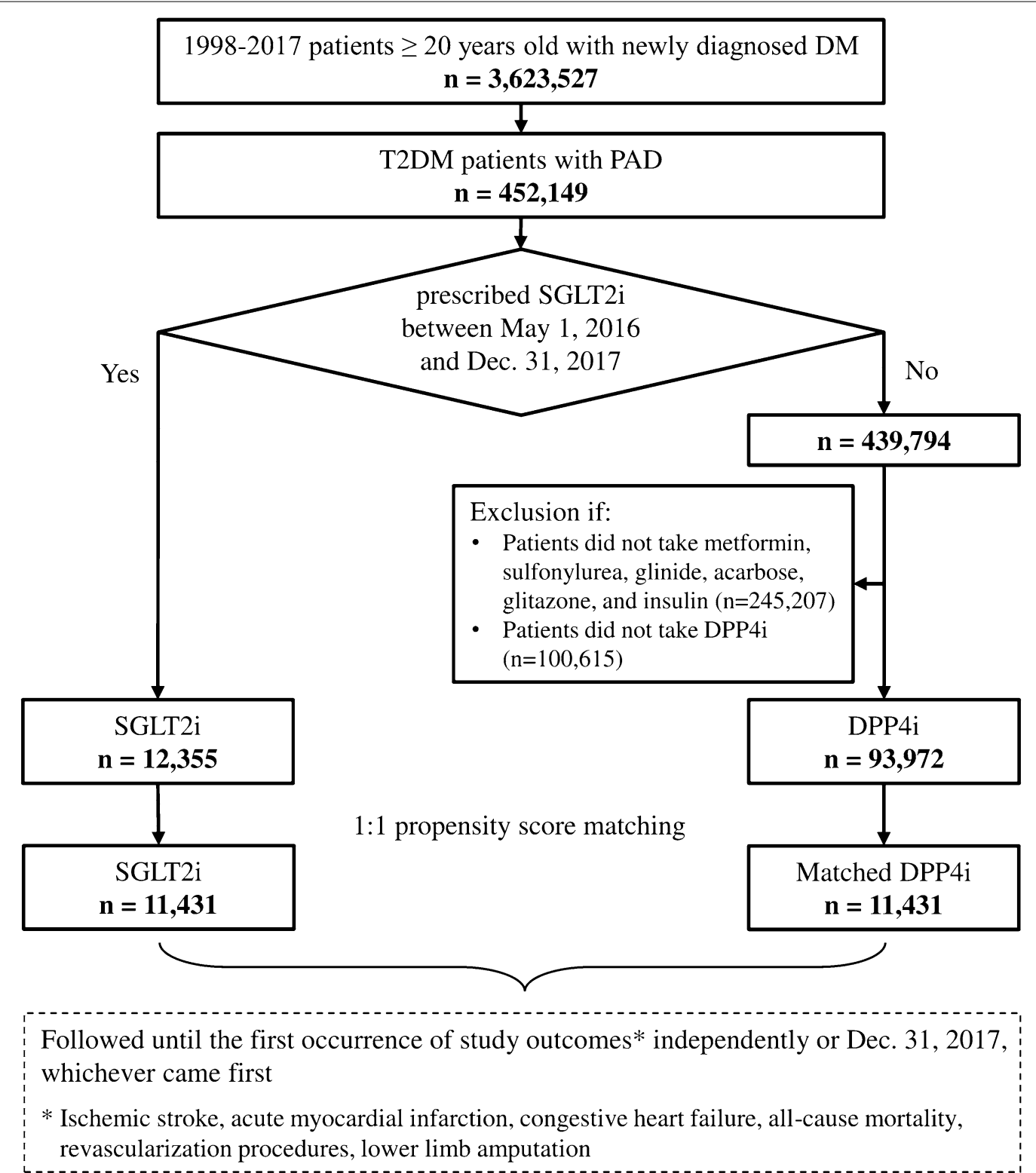

Fig. 1 Enrollment of patients with concomitant type-2 diabetes mellitus (T2DM) and peripheral artery disease (PAD). From May 1, 2016 to December 31, 2017, a total of 11,431 patients with T2DM and comorbid PAD treated with sodium-glucose co-transporter-2 inhibitors (SGLT2i) and 11,431 1:1 propensity score matched patients treated with dipeptidyl peptidase-4 inhibitors (DPP4i) were enrolled in the present study. Abbreviations: DPP4i dipeptidyl peptidase-4 inhibitor, PAD peripheral artery disease; SGLT2i sodium-glucose co-transporter-2 inhibitor, T2DM type 2 diabetes mellitus

\section{Covariates and study outcomes}

Baseline covariates were obtained from all claim records with diagnoses, procedures, or medication codes prior to the index date. A history of all prescription medications was confined to medications used at least once within 3 months before the index date. We reported the following outcomes in the present study: (i) ischemic stroke (IS), (ii) acute myocardial infarction (AMI), (iii) congestive heart failure (CHF), (iv) lower limb ischemia requiring revascularization, (v) lower limb amputation, (vi) all-cause mortality, and (vii) cardiovascular mortality. All study outcomes should be the primary discharge diagnosis to avoid misclassification. The diagnostic codes of the NHIRD were shifted from the ICD-9-CM to ICD10-CM after January 1, 2016. The ICD-9-CM and ICD$10-\mathrm{CM}$ codes used to identify study outcomes along with the baseline covariates are summarized in Additional file 1: Tables S1 and S2. 


\section{Statistical analysis}

The propensity score matching (PSM) method, which simulates the design of a randomized clinical trial for observational cohort data by forming matched sets of treated and untreated subjects who share a similar value of the propensity score [16], was used to compare the study outcomes between the SGLT2i and DPP4i group. We calculated propensity score, the predicted probability of treatment conditional on all the covariates in Table 1, by using the generalized boosted model (GBM). The GBM involves an iterative process with multiple regression trees to capture complex and nonlinear relationships between treatment assignment and the pretreatment covariates without over-fitting the data and leading the best balance across study groups [17]. The PSM ratio between the SGLT2i users and DPP4i users was 1:1 without replacement and nearest neighbor matching within a caliper width (8-to-1 digit matching) [18]. The balance of potential confounders at the baseline (index date) between study groups was assessed using the absolute standardized mean difference (ASMD) rather than statistical testing because balance is a property of the sample and not of the underlying population. An ASMD value of $\leq 0.1$ would indicate a nonsignificant difference in potential confounders between the two study groups [19]. Incidence rates were estimated using the total number of study outcomes during the follow-up period divided by person-years at risk. The risk of study outcomes occurring over the follow-up duration for SGLT2i versus DPP4i (reference) was obtained using survival analysis (Kaplan-Meier method and log-rank test for univariate analysis and Cox proportional hazards model for multivariate analysis), and they were presented as HRs with 95\% CIs. Statistical significance was defined as a $p$ value of $<0.05$. All statistical analyses were performed using SAS 9.4 (SAS Institute Inc., Cary, NC, USA).

\section{Results}

\section{Baseline characteristics of SGLT2i and DDP4i groups}

Among the 452,149 patients with T2DM and concomitant PAD, a total of 12,355 and 93,972 were treated with SGLT2i and DDP4i, respectively, from May 1, 2016 to December 31, 2017 (Fig. 1). The mean follow-up periods were $0.96 \pm 0.57$ and $0.66 \pm 0.45$ years for SGLT2i and DDP4i, respectively. In the SGLT2i group, 6,915 (56.0\%) and 5,440 (44.0\%) patients were treated with dapagliflozin and empagliflozin, respectively. In the DDP4i group, 29,782 (31.7\%), 24,833 (26.4\%), 28,534 (30.4\%), 10,636 (11.3\%), and $187(0.2 \%)$ patients were treated with sitagliptin, vildagliptin, linagliptin, saxagliptin, and alogliptin, respectively. Before PSM, the SGLT2i group was younger and had a lower prevalence of chronic kidney disease (CKD), hypertension, CHF, hypertension, and stroke history compared with the DDP4i group. The SGLT2i group had a higher rate of dyslipidemia, higher rate of prescriptions for statins, metformin, sulfonylurea, acarbose, and glitazones and a lower rate of prescriptions for glinides. Both study groups were well balanced in all characteristics after PSM (all ASMD <0.1) (Table 1).

\section{Main analysis of SGLT2i versus DDP4i}

The SGLT2i group had comparable cumulative risks of IS and AMI compared with the DPP4i group after PSM. The SGLT2i group was associated with a lower cumulative risk of CHF (log-rank $p=0.0059)$, all-cause (logrank $p<0.0001)$ and cardiovascular mortality (log-rank $p=0.0085)$ compared with the DPP4i group after PSM. Regarding major adverse limb events, the use of SGLT2i was associated with a lower cumulative risk of lower limb revascularization (log-rank $p=0.0359)$ and amputation (log-rank $p<0.0001)$ compared with the use of DPP4i (Fig. 2).

The incidence rates (per 100 person-years) of IS (1.26 vs. $1.54, p=0.1213$ ) and AMI (0.66 vs. $0.77, p=0.3702$ ) were comparable between the SGLT2i and DDP4i groups. The SGLT2i group had a significantly lower incidence rate of CHF (0.96 vs. 1.43; HR: 0.66; $95 \% \mathrm{CI}$ $0.49-0.89 ; p=0.0062)$, lower limb ischemia requiring revascularization $(0.97$ vs. 1.32 ; HR: 0.73 ; $95 \%$ CI $0.54-$ $0.98 ; p=0.0367)$, lower limb amputation (0.54 vs. 1.23 ; HR: 0.43; 95\% CI: 0.30-0.62; $p<0.0001$ ), all-cause mortality ( 3.19 vs. 5.44 , HR: 0.58 ; $95 \%$ CI $0.49-0.67 ; p<0.001$ ), and cardiovascular mortality (0.91 vs. 1.33 , HR: 0.67 ; 95\% CI 0.49-0.90; $p=0.0089$ ) compared with the DDP4i group (Table 2 and Fig. 3). The use of SGLT2i was not associated with an increased risk of bone fracture or urinary tract infection compared with DDP4i use after PSM (Table 2 and Additional file 1: Table S1).

\section{Subgroup analysis of high-risk patients}

The subgroup analysis indicated that SGLT2i reduced the risk of AMI in patients with concomitant CKD but not in those without CKD ( $p$ interaction $=0.02$; Fig. 4 ). In general, the subgroup analysis revealed consistent results for CHF, major adverse limb outcomes, and mortality for SGLT2i versus DPP4i among patients aged $\geq 75$ years, the presence of $C K D$ and established $C V$ disease, consistent with the main analysis (Figs. 4, 56 ).

\section{Discussion}

To the best of our knowledge, the present study is the first and largest population-based cohort study to investigate the outcomes of patients with T2DM and concomitant PAD treated with SGLT2i compared with those treated with DPP4i. Our findings indicate that SGLT2i was associated with comparable risks of IS and AMI, 
Table 1 Clinical characteristics of patients with concomitant type-2 diabetes mellitus (T2DM) and peripheral artery disease (PAD) treated with sodium-glucose co-transporter-2 inhibitors (SGLT2i) and dipeptidyl peptidase-4 inhibitors (DPP4i) before and after propensity score matching (PSM)

\begin{tabular}{|c|c|c|c|c|c|c|}
\hline & \multicolumn{3}{|l|}{ Before PSM } & \multicolumn{3}{|l|}{ After PSM } \\
\hline & $\begin{array}{l}\text { SGLT2i } \\
(n=12,355)\end{array}$ & $\begin{array}{l}\text { DPP4i } \\
(n=93,972)\end{array}$ & ASMD & $\begin{array}{l}\text { SGLT2i } \\
(n=11,431)\end{array}$ & $\begin{array}{l}\text { DPP4i } \\
(n=11,431)\end{array}$ & ASMD \\
\hline \multicolumn{7}{|l|}{ Baseline characteristics } \\
\hline \multicolumn{7}{|l|}{ Age (years) } \\
\hline Mean & $64.3 \pm 10.6$ & $70.5 \pm 11.3$ & 0.5639 & $64.7 \pm 10.7$ & $65.1 \pm 14.5$ & 0.0331 \\
\hline$<65$ & $6188(50.08 \%)$ & $27900(29.69 \%)$ & 0.5385 & $5487(48.00 \%)$ & $5662(49.53 \%)$ & 0.0716 \\
\hline $65-74$ & 3990 (32.29\%) & $29360(31.24 \%)$ & & $3789(33.15 \%)$ & $3677(32.17 \%)$ & \\
\hline $75-84$ & $1861(15.06 \%)$ & $26506(28.21 \%)$ & & 1839 (16.09\%) & $1791(15.67 \%)$ & \\
\hline$\geqq 85$ & $316(2.56 \%)$ & $10206(10.86 \%)$ & & $316(2.76 \%)$ & 301 (2.63\%) & \\
\hline Male & 6167 (49.92\%) & $44738(47.61 \%)$ & 0.0462 & $5603(49.02 \%)$ & $5660(49.51 \%)$ & 0.0100 \\
\hline Chronic lung disease & $376(3.04 \%)$ & 3852 (4.10\%) & 0.0569 & 339 (2.97\%) & 344 (3.01\%) & 0.0026 \\
\hline Chronic kidney disease & $3276(26.52 \%)$ & $37701(40.12 \%)$ & 0.2917 & 3117 (27.27\%) & $2997(26.22 \%)$ & 0.0237 \\
\hline Congestive heart failure & 407 (3.29\%) & $5279(5.62 \%)$ & 0.1128 & $354(3.10 \%)$ & 351 (3.07\%) & 0.0015 \\
\hline Hypertension & $10530(85.23 \%)$ & $84307(89.72 \%)$ & 0.1358 & $9779(85.55 \%)$ & $9730(85.12 \%)$ & 0.0121 \\
\hline Dyslipidemia & $11062(89.53 \%)$ & $80720(85.90 \%)$ & 0.1110 & $10208(89.30 \%)$ & $10199(89.22 \%)$ & 0.0025 \\
\hline Previous stroke & $1159(9.38 \%)$ & $14043(14.94 \%)$ & 0.1708 & 1102 (9.64\%) & $1.036(9.06 \%)$ & 0.0198 \\
\hline Ischemic heart disease & 2595 (21.00\%) & $18827(20.03 \%)$ & 0.0240 & $2176(19.04 \%)$ & $2172(19.00 \%)$ & 0.0009 \\
\hline Gout & 3823 (30.94\%) & $32206(34.27 \%)$ & 0.0711 & $3562(31.16 \%)$ & $3532(30.90 \%)$ & 0.0057 \\
\hline Malignancy & $826(6.69 \%)$ & 8644 (9.20\%) & 0.0930 & $776(6.79 \%)$ & $782(6.84 \%)$ & 0.0021 \\
\hline History of bleeding & $96(0.78 \%)$ & $1639(1.74 \%)$ & 0.0868 & 95 (0.83\%) & $86(0.75 \%)$ & 0.0089 \\
\hline $\mathrm{PCl}$ & $1570(12.71 \%)$ & $11113(11.83 \%)$ & 0.0269 & 1277 (11.17\%) & $1256(10.99 \%)$ & 0.0059 \\
\hline CABG & $296(2.40 \%)$ & $2547(2.71 \%)$ & 0.0199 & $253(2.21 \%)$ & $267(2.34 \%)$ & 0.0082 \\
\hline History of diabetic ulcer & $161(1.30 \%)$ & $2117(2.25 \%)$ & 0.0719 & $148(1.29 \%)$ & $183(1.60 \%)$ & 0.0256 \\
\hline \multicolumn{7}{|l|}{ Baseline medications } \\
\hline Use of APT & $5506(44.56 \%)$ & $41766(44.45 \%)$ & 0.0024 & $4969(43.47 \%)$ & 4903 (42.89\%) & 0.0117 \\
\hline Use of NSAIDs & $3516(28.46 \%)$ & $25954(27.62 \%)$ & 0.0187 & $3299(28.86 \%)$ & $3260(28.52 \%)$ & 0.0075 \\
\hline Use of PPI & 859 (6.95\%) & 8454 (9.00\%) & 0.0755 & $820(7.17 \%)$ & 781 (6.83\%) & 0.0134 \\
\hline Use of ACEI/ARB & 7970 (64.51\%) & $57265(60.94 \%)$ & 0.0739 & 7299 (63.85\%) & 7305 (63.91\%) & 0.0011 \\
\hline Use of amiodarone & $202(1.63 \%)$ & $2564(2.73 \%)$ & 0.0749 & $197(1.72 \%)$ & 205 (1.79\%) & 0.0053 \\
\hline Use of dronedarone & $14(0.11 \%)$ & $145(0.15 \%)$ & 0.0112 & $9(0.08 \%)$ & $13(0.11 \%)$ & 0.0113 \\
\hline Use of beta-blocker & $4703(38.07 \%)$ & 34399 (36.61\%) & 0.0302 & $4222(36.93 \%)$ & $4166(36.44 \%)$ & 0.0102 \\
\hline Use of verapamil/diltiazem & $688(5.57 \%)$ & $5454(5.80 \%)$ & 0.0102 & $615(5.38 \%)$ & $601(5.26 \%)$ & 0.0055 \\
\hline Use of digoxin & 247 (2.00\%) & 2125 (2.26\%) & 0.0182 & $220(1.92 \%)$ & $220(1.92 \%)$ & 0.0000 \\
\hline Use of statin & $8469(68.55 \%)$ & $51472(54.77 \%)$ & 0.2862 & 7609 (66.56\%) & 7651 (66.93\%) & 0.0078 \\
\hline Use of metformin & $6910(55.93 \%)$ & $33242(35.37 \%)$ & 0.4217 & $6403(56.01 \%)$ & $6484(56.72 \%)$ & 0.0143 \\
\hline Use of sulfonylurea & $8282(67.03 \%)$ & $50171(53.39 \%)$ & 0.2815 & $7630(66.75 \%)$ & $7751(67.81 \%)$ & 0.0226 \\
\hline Use of glinide & $811(6.56 \%)$ & $11886(12.65 \%)$ & 0.2076 & 785 (6.87\%) & $753(6.59 \%)$ & 0.0112 \\
\hline Use of acarbose & $2194(17.76 \%)$ & $12488(13.29 \%)$ & 0.1236 & $1889(16.53 \%)$ & $1870(16.36 \%)$ & 0.0045 \\
\hline Use of glitazone & $2617(21.18 \%)$ & $9538(10.15 \%)$ & 0.3071 & $2244(19.63 \%)$ & $2305(20.16 \%)$ & 0.0134 \\
\hline Use of insulin & 3841 (31.09\%) & $26103(27.78 \%)$ & 0.0727 & 3433 (30.03\%) & 3326 (29.10\%) & 0.0205 \\
\hline Use of loop diuretics & $1286(10.41 \%)$ & 14791 (15.74\%) & 0.1586 & 1191 (10.42\%) & 1170 (10.24\%) & 0.0060 \\
\hline Use of MRA & $663(5.37 \%)$ & 5025 (5.35\%) & 0.0008 & $592(5.18 \%)$ & $617(5.40 \%)$ & 0.0098 \\
\hline Use of ARNI & $16(0.13 \%)$ & $28(0.03 \%)$ & 0.0353 & $7(0.06 \%)$ & $10(0.09 \%)$ & 0.0096 \\
\hline
\end{tabular}

ACEl angiotensin-converting-enzyme inhibitor, $A P T$ antiplatelet agent, $A R B$ angiotensin II receptor antagonists, $A R N /$ angiotensin receptor neprilysin inhibitor, $A S M D$ absolute standardized mean difference, $C A B G$ coronary artery bypass graft, $D D P 4$ idipeptidyl peptidase-4 inhibitors, DM diabetes mellitus, $M R A$ mineralocorticoid receptor antagonist, NSAIDs nonsteroid anti-inflammatory drugs, $P A D$ peripheral artery disease, $P C /$ percutaneous coronary intervention, $P P /$ proton pump inhibitor, PSM propensity score matching, SGLT2i sodium-glucose co-transporter-2 inhibitors, T2DM type-2 diabetes mellitus 


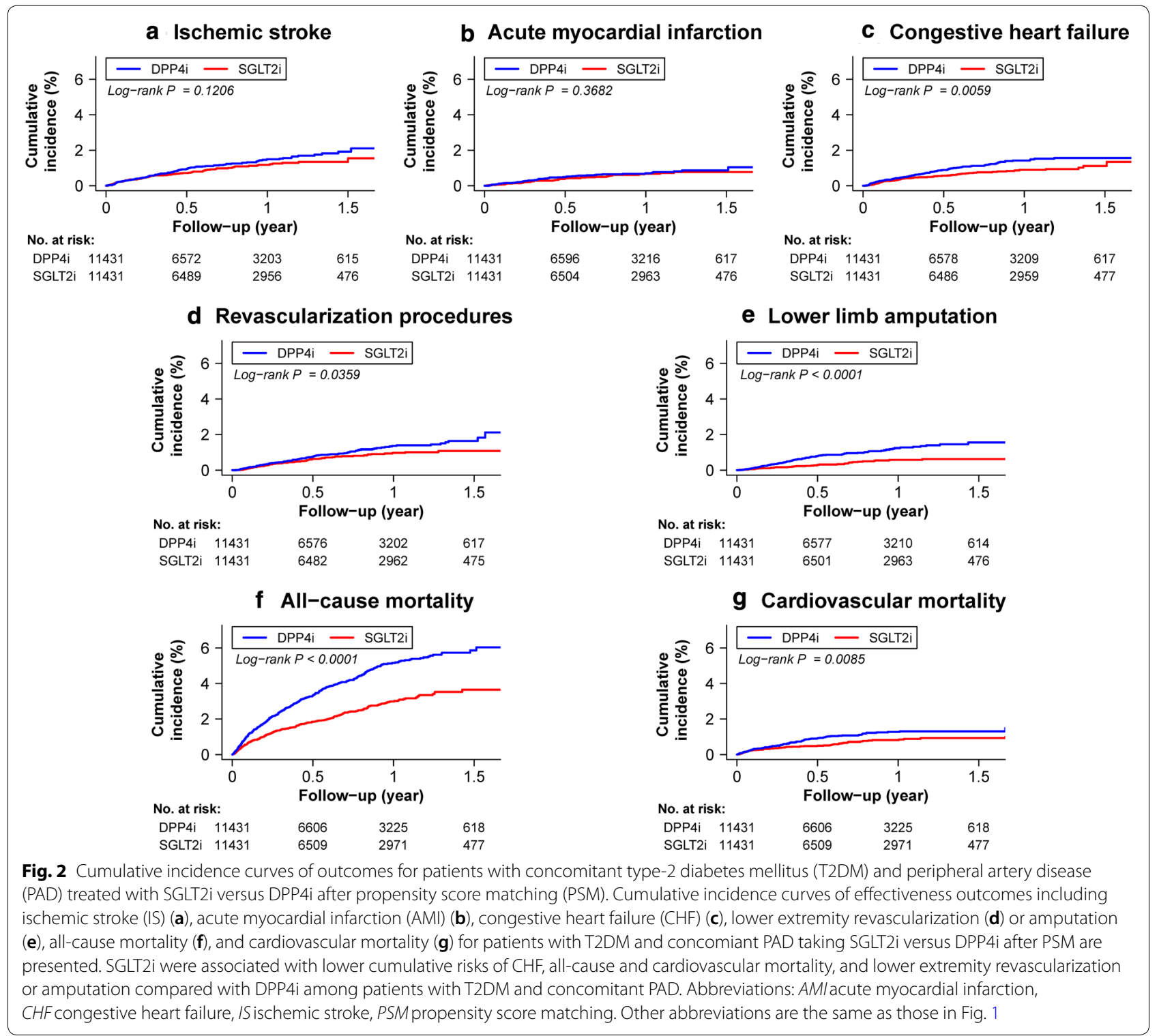

and significantly lower risks of CHF, lower limb ischemia requiring revascularization or amputation, and all-cause or cardiovascular mortality when compared with DPP4i. This study suggests that SGLT2i is an effective and safe alternative to DPP4i for patients with T2DM and concomitant PAD.

DPP4i improves glycemic control by increasing the serum levels of glucagon-like peptide 1 (GLP-1) through the inhibition of GLP-1 degradation, which indirectly stimulates insulin secretion and enhances beta-cell function. Previous large-scale clinical trials, including EXAMINE, SAVOR-TIMI53, and TECOS, have indicated that the use of DPP4i has a neutral effect in CV composite outcomes for patients with T2DM, except for a higher risk of $\mathrm{CHF}$ for those treated with saxagliptin [20-22]. Those clinical studies did not explore the risk of lower limb outcomes for patients with T2DM treated with DPP4i. A previous meta-analysis of the three clinical trials confirmed the benefit of SGLT2i on CHF (HR 0.69; 95\% CI 0.61-0.79), all-cause death (HR 0.85; 95\% CI 0.78-0.93), and reduced risk of major adverse CV events (composite of myocardial infarction, stroke, and cardiovascular death; HR 0.89, CI 0.83-0.96]) [23]. One large retrospective cohort study also indicated that SGLT2i were associated with lower risks of $\mathrm{CHF}$ and death compared with DPP4i in patients with diabetes [24]. Other cohort studies investigating SGLT2i versus other nonSGLT2i antidiabetic agents have consistently reported 
Table 2 Number of events, event rates, and hazard ratio (HR) among patients with type-2 diabetes mellitus and concomitant peripheral artery disease (PAD) using sodium-glucose co-transporter-2 inhibitors (SGLT2i) versus dipeptidyl peptidase-4 inhibitors (DPP4i) after propensity score matching

\begin{tabular}{|c|c|c|c|c|c|c|}
\hline \multirow[b]{3}{*}{ Clinical outcome } & \multirow{2}{*}{\multicolumn{2}{|c|}{$\begin{array}{l}\text { SGLT2i } \\
(n=11,431)\end{array}$}} & \multirow{2}{*}{\multicolumn{2}{|c|}{$\begin{array}{l}\text { DPP4i } \\
(n=11,431)\end{array}$}} & \multirow{2}{*}{\multicolumn{2}{|c|}{ Cox model }} \\
\hline & & & & & & \\
\hline & Number & $\begin{array}{l}\text { Incidence rate (per } \\
100 \mathrm{PYs} \text { ) }\end{array}$ & Number & $\begin{array}{l}\text { Incidence rate (per } \\
100 \mathrm{PYs} \text { ) }\end{array}$ & $\mathrm{HR}(95 \% \mathrm{Cl})$ & $p$ value \\
\hline Ischemic stroke (IS) & 96 & 1.26 & 120 & 1.54 & $0.81(0.62-1.06)$ & 0.1213 \\
\hline Acute myocardial infarction (AMI) & 50 & 0.66 & 60 & 0.77 & $0.84(0.58-1.23)$ & 0.3702 \\
\hline Congestive heart failure (CHF) & 73 & 0.96 & 111 & 1.43 & $0.66(0.49-0.89)$ & 0.0062 \\
\hline $\begin{array}{l}\text { Lower limb ischemia requiring revas- } \\
\text { cularization }\end{array}$ & 74 & 0.97 & 103 & 1.32 & $0.73(0.54-0.98)$ & 0.0367 \\
\hline Lower limb amputation & 41 & 0.54 & 96 & 1.23 & $0.43(0.30-0.62)$ & $<0.0001$ \\
\hline All-cause mortality & 243 & 3.19 & 425 & 5.44 & $0.58(0.49-0.67)$ & $<0.0001$ \\
\hline Cardiovascular mortality & 69 & 0.91 & 104 & 1.33 & $0.67(0.49-0.90)$ & 0.0089 \\
\hline \multicolumn{7}{|l|}{ Safety outcome } \\
\hline Urinary tract infection & 331 & 4.42 & 297 & 3.87 & $1.13(0.96-1.32)$ & 0.1367 \\
\hline Bone fracture & 76 & 1.00 & 71 & 0.91 & $1.08(0.78-1.50)$ & 0.6284 \\
\hline
\end{tabular}

AMI acute myocardial infarction, CHF congestive heart failure, $C l$ confidence interval, DDP4i dipeptidyl peptidase-4 inhibitors, $H R$ hazard ratio, IS ischemic stroke, $P A D$ peripheral artery disease, $P S M$ propensity score matching, $P Y$ s person-years, SGLT2i sodium-glucose co-transporter-2 inhibitors, $T 2 D M$ type-2 diabetes mellitus

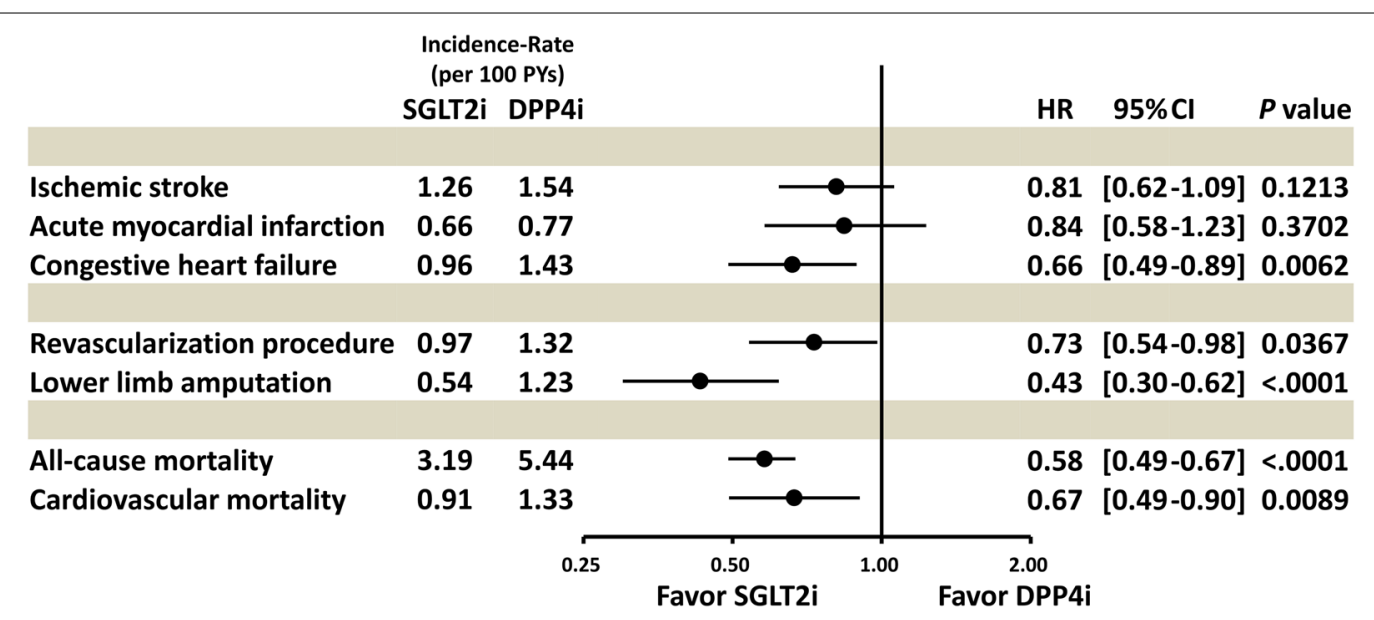

Fig. 3 Forest plot of the hazard ratios of clinical outcomes for SGLT2i versus DPP4i among patients with type-2 diabetes mellitus (T2DM) comorbid with peripheral artery disease (PAD) after propensity score matching (PSM). SGLT2i were associated with a comparable risk of thromboembolic events and with lower risks of CHF, lower limb revascularization or amputation, and all-cause or cardiovascular mortality compared with DPP4i among patients with T2DM and concomitant PAD after PSM. Abbreviation: Clconfidence interval, HR hazard ratio. Other abbreviations are the same as those in Figs. 1, 2

that SGLT2i reduces the risk of CHF [25-27]. A recent clinical trial also showed the beneficial role of SGLT2i on reducing the risk of HF hospitalization [28, 29].

Patients with T2DM have a higher prevalence of PAD compared with those without T2DM, and patients with T2DM and concomitant PAD have a higher risk of mortality and amputation [11,30]. However, evidence supporting the benefits of SGLT2i in patients with diabetes and concomitant PAD is limited. The CANVAS program reported a higher rate of amputations in the canagliflozin group compared with the placebo group ( 0.63 vs. 0.34 per 100 person-years, $p<0.001$ ), but not in the pivot studies of empagliflozin (0.65 vs. 0.65 per 100 person-years, $p=1.000)$ and dapagliflozin (1.4 vs. 1.3 per 100 personyears, $p=0.53)$ [1-3]. A clear mechanism explaining why canagliflozin contributes to amputation is lacking; this adverse event may be related to volume depletion, which might accordingly cause circulatory failure in the 


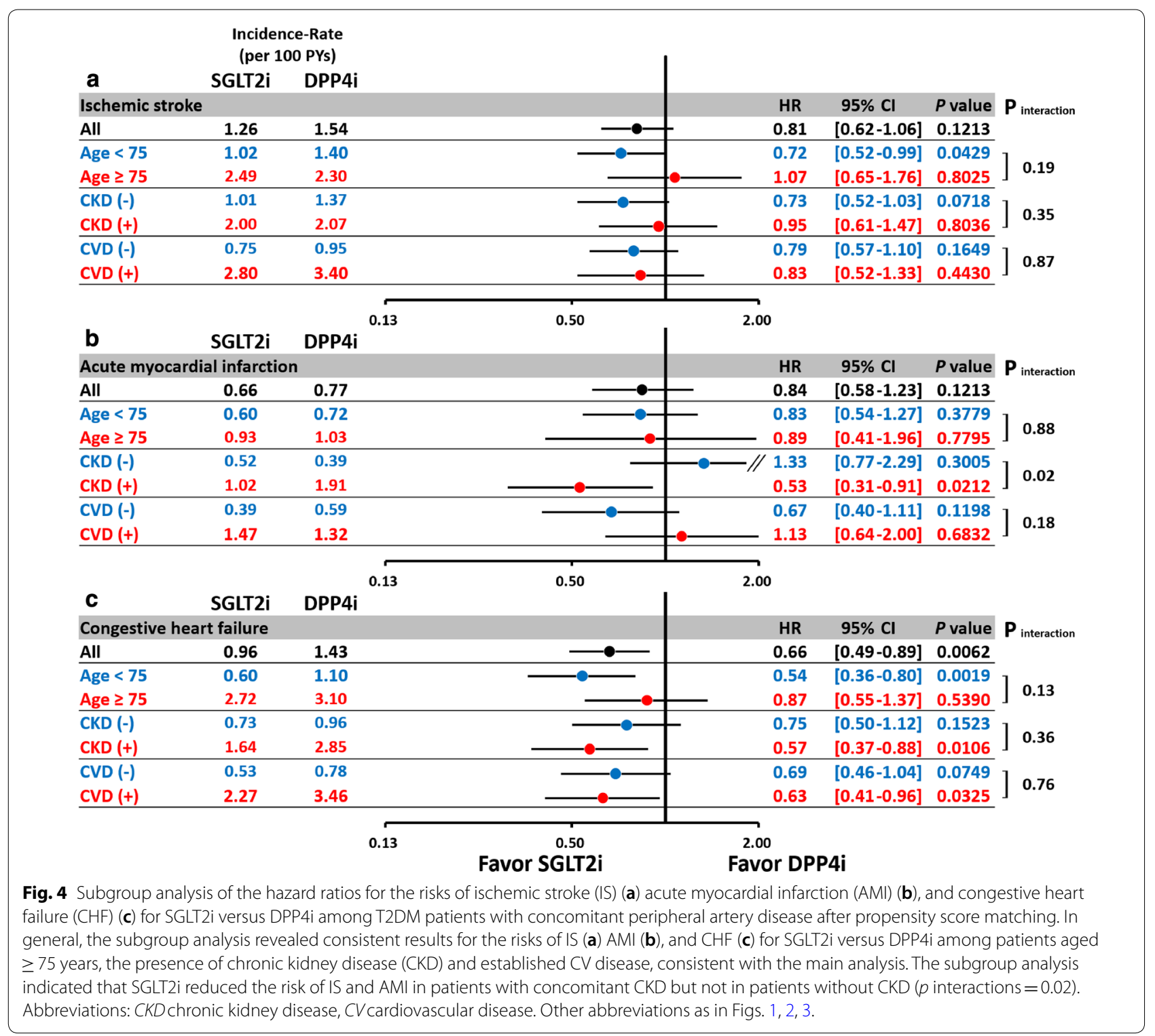

distal peripheral vasculature $[1,31]$. Although a metaanalysis showing patients treated with SGLT2i without a significant association with increased risk of amputation, a large-scale cohort study revealed that SGLT2is were associated with an increased risk of amputation compared with other antihyperglycemic agents for type 2 diabetes [32, 33]. Subgroup analyses from the pivot study of empagliflozin for patients with T2DM and concomitant PAD also revealed benefits of reduced risks of death and CHF without an increased risk of amputation [12]. Consistent with the data, our results indicated that SGLT2i can reduce the risks of CHF and mortality in such a high-risk population. Notably, SGLT2i (dapagliflozin and empagliflozin) were associated with a lower risk of adverse limb events (lower limb ischemia requiring revascularization and lower limb amputation) compared with DDP4i in our study (0.97 vs. 1.32 per 100 personyears, $p=0.0367$ and 0.54 vs. 1.23 per 100 person-years, $p<0.0001)$. In the assessment of patients with T2DM and concomitant PAD with a relatively high risk of amputation, the absolute risk of amputation in patients treated with SGLT-2i was similar or lower than those seen in the pivot studies, and there is no increase in the probability of amputation [1-3]. In animal or human studies, SGLT2i have been reported to have many benefits for vasculature, such as improved endothelial function, vasodilatation, and attenuated oxidative stress, suggesting that SGLT2i may be able to halt the progression of atherosclerosis and 


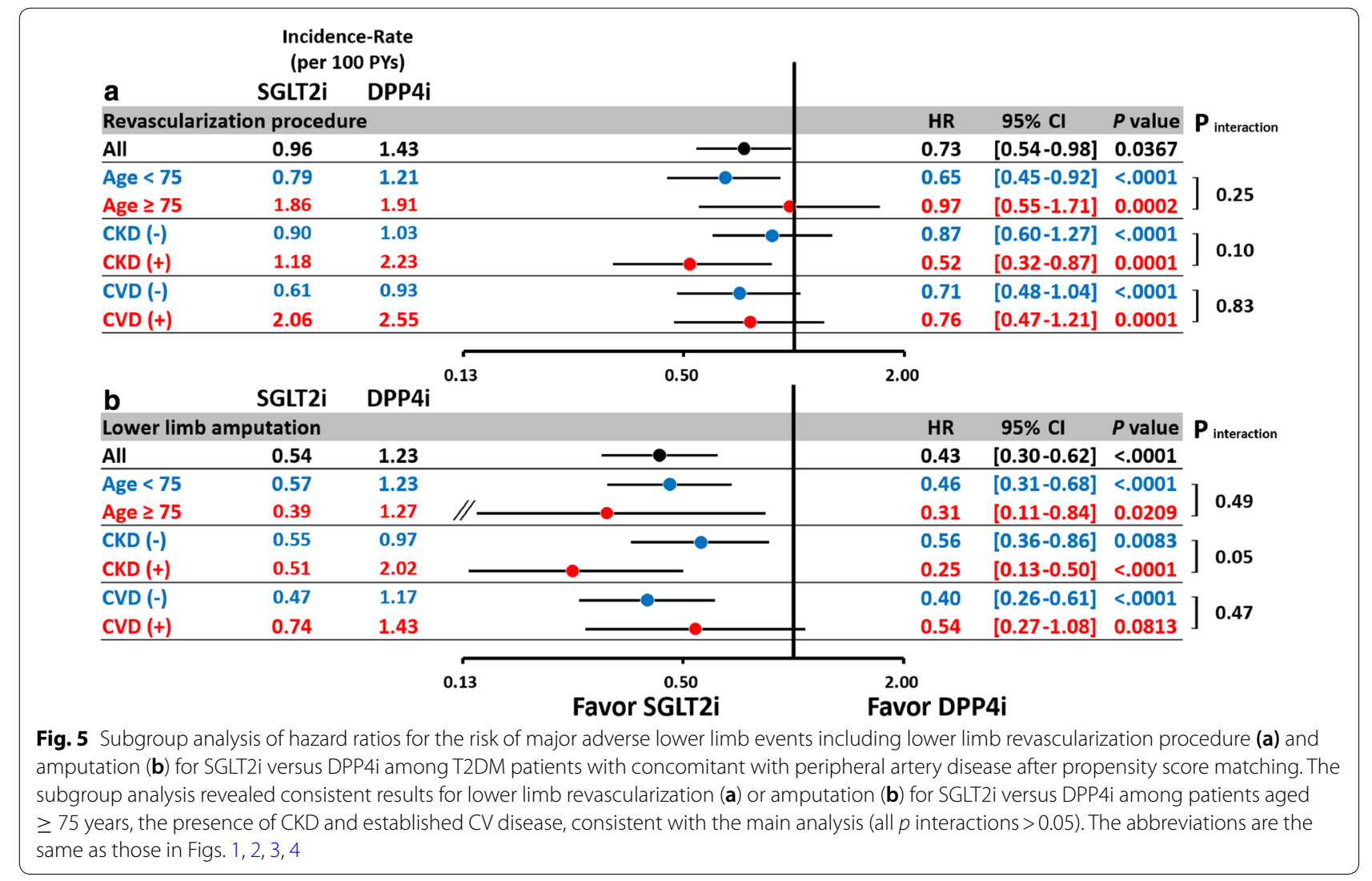

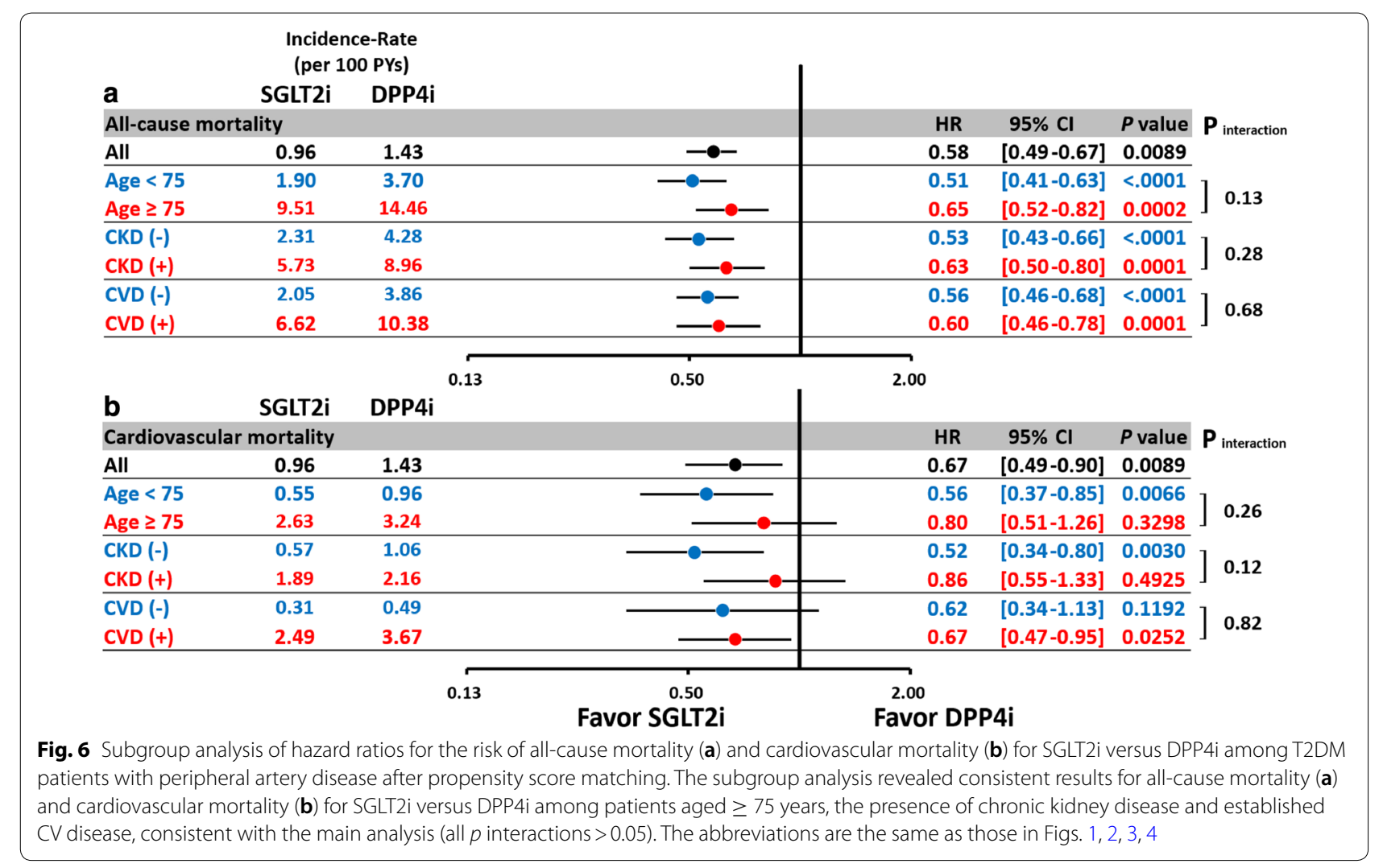


improve vascular outcomes [34-36]. In addition, SGLT2i had been reported to improve cardiometabolic risk factors than DDP4i [37]. PAD is a manifestation of systemic atherosclerosis, and because SGLT2i could reduce the risk of adverse atherosclerotic events, it may also be beneficial in reducing the risk of adverse limb events for patients with PAD [23, 38, 39]. However, studies investigating SGLT2i in patients with T2DM and concomitant PAD are scarce. A subgroup analysis revealed a trend of a lower risk of lower limb amputation (HR: 0.84, 95\% CI 0.54-1.32) in the empagliflozin group among patients with T2DM and concomitant PAD [12]. Because patients with T2DM have a high prevalence of PAD [8-10], further randomized or prospective studies should investigate the effect of SGLT2i on lower limb outcomes in such a high-risk population.

\section{Limitations}

To avoid time-lag bias from the prescriptions of study drugs, which may lead to false positive or negative associations depending on the treatments for patients with early or advanced disease, we selected the same secondline hypoglycemic agents of DDP4i as the comparator in our study [7, 40]. To avoid immortal time bias, our study only included new descriptions of study drugs of SGLT2i or DDP4i without baseline use [40, 41]. Nevertheless, the present study had several limitations. First, although PSM with several variables allowed the matching of baseline comorbidities among the study groups, residual confounding by unmeasured variables and prescribing behavior could not be excluded in this retrospective cohort study. Second, the NHIRD does not contain several crucial types of laboratory data such as body weight, glycohemoglobin (HbA1c), and serum creatinine, all of which are associated with the risk of $\mathrm{CV}$ events and death among patients with T2DM [42]. In addition, even with adjustment for CKD, the diagnosis of CKD by coding could not reflect the severity of renal disease, which may interfere with SGLT2i or DDP4i selection for each patient. Third, although we utilized some criteria for the selection of the PAD population, our PAD study patients included only part of the PAD population. Thoroughly screening patients with PAD is difficult because PAD populations are typically underrecognized or undertreated in clinical practice, and the incidence of asymptomatic PAD is higher than that of symptomatic PAD [43, 44]. Fourth, miscoding and misclassification of underlying comorbidities and outcomes registered by each physician were another limitation. Therefore, we only considered primary discharge diagnoses to improve the outcome accuracy. However, minor cardiovascular or limb events without admission may have been missed in the present study. Fifth, we did not analyze canagliflozin because of its approval date after March 1, 2018 in Taiwan. Finally, we only investigated Asian patients, and whether our results can be extrapolated to other races remains unclear.

\section{Conclusions}

Our data indicated that SGLT2i, compared with DDP4i, were associated with lower risks of CHF, lower limb ischemia requiring revascularization or amputation, and all-cause death for patients with T2DM and concomitant PAD. Further prospective studies are necessary to evaluate the effects of SGLT2i on lower limb outcomes among such patients in the future.

\section{Supplementary information}

Supplementary information accompanies this paper at https://doi. org/10.1186/s12933-020-01118-0.

Additional file 1: Table S1. International Classification of Diseases (9th and 10th edition) Clinical Modification (ICD 9-CM and ICD 10-CM) codes used to define comorbidities and clinical outcomes in this study. Table S2. International Classification of Diseases (9th and 10th edition) Clinical Modification (ICD 9-CM and ICD 10-CM) codes used to define major adverse limb outcomes in this study. Table S3. Number of events, event rates, and hazard ratio (HR) among patients with type-2 diabetes mellitus concomitant with peripheral artery disease using sodium-glucose cotransporter-2 inhibitors (SGLT2i) versus dipeptidyl peptidase-4 inhibitors (DPP4i) before propensity score matching.

\section{Abbreviations}

ACEl: Angiotensin-converting enzyme inhibitor; AMI: Acute myocardial infarction; APT: Antiplatelet agent; ARB: Angiotensin receptor blocker; ARNI: Angiotensin receptor-neprilysin inhibitor; ASMD: Absolute standardized mean difference; CABG: Coronary artery bypass graft; $\mathrm{CHF}$ : Congestive heart failure; Cl: Confidence interval; CKD: Chronic kidney disease; DPP4i: Dipeptidyl peptidase-4 inhibitor; HR: Hazard ratio; IS: Ischemic stroke; MRA: Mineralocorticoid receptor antagonist; NSAIDs: Nonsteroid anti-inflammatory drugs; PAD: Peripheral artery disease; PCI: Percutaneous coronary intervention; PPI: Proton pump inhibitor; PSM: Propensity score matching; PYs: Person-years; SGLT2i: Sodium glucose cotransporter-2 inhibitor; T2DM: Type-2 diabetes mellitus.

\section{Acknowledgements}

This study was based in part on the National Health Insurance Research Database provided by the Health and Welfare Data Center (HWDC) from Ministry of Health and Welfare (MOHW), Taiwan. The interpretation and conclusions contained herein do not represent those of the National Health Insurance Administration, Ministry of Health and Welfare, Taiwan.

\section{Authors' contributions}

HFL and YHC contributed to the conception and design of the study as well as the analysis and interpretation of the data, and they wrote the manuscript and approved its submission. PRL, JRL, and LCS contributed to data acquisition and analysis. YHC and LCS contributed to the analysis of data and provided critical revisions. SWC, LSW, SHC, YHY, CTK, and LCS contributed to the conception and design, and they provided critical revisions of the paper for crucial intellectual content. All authors read and approved the final manuscript.

\section{Funding}

This study was supported by grant 108-2314-B-182-053-MY2 from Ministry of Science and Technology, Taiwan, and grants CMRPG3J1371, CMRPD1K0031, CMRPG3K0021, CMRPG3J0841, and CORPG3G0351, CLRPG3D0046 from Chang Gung Memorial Hospital, Linkou, Taiwan. 


\section{Availability of data and materials}

The datasets used in this study were only available from the Health and Welfare Data Center, Taiwan. The SAS programs (codes) involved in this study are available from the corresponding author upon reasonable request.

\section{Ethics approval and consent to participate}

The study protocol complied with the Declaration of Helsinki and was approved by the Institutional Review Board of the Chang Gung Medical Foundation, Taiwan.

\section{Consent for publications}

Not applicable.

\section{Competing interests}

The authors declare that they have no competing interests.

\section{Author details}

${ }^{1}$ The Cardiovascular Department, Chang Gung Memorial Hospital, Linkou, Taoyuan 33305, Taiwan. ${ }^{2}$ College of Medicine, Chang Gung University, Taoyuan 33302, Taiwan. ${ }^{3}$ Graduate Institute of Clinical Medical Sciences, College of Medicine, Chang Gung University, Taoyuan, Taiwan. ${ }^{4}$ Division of Cardiology, Department of Internal Medicine, New Taipei City Municipal Tucheng Hospital (Chang Gung Memorial Hospital, Tucheng branch, Taiwan), Taoyuan, Taiwan. ${ }^{5}$ Division of Thoracic and Cardiovascular Surgery, Department of Surgery, Linkou Medical Center, Chang Gung Memorial Hospital, Chang Gung University, Taoyuan City, Taiwan. ${ }^{6}$ Department of Public Health, College of Medicine, Chang Gung University, No. 259, Wenhua 1st Rd., Guishan Dist, Taoyuan 33302, Taiwan. ${ }^{7}$ Center for Big Data Analytics and Statistics, Chang Gung Memorial Hospital, Taoyuan, Taiwan. ${ }^{8}$ Microscopy Core Laboratory, Chang Gung Memorial Hospital, Linkou, Taoyuan 33305, Taiwan. ${ }^{9}$ Biostatistics Core Laboratory, Molecular Medicine Research Center, Chang Gung University, Taoyuan 33302, Taiwan. ${ }^{10}$ Division of Rheumatology, Allergy and Immunology, Department of Internal Medicine, Chang Gung Memorial Hospital, Linkou, Taoyuan 33305, Taiwan.

\section{Received: 2 July 2020 Accepted: 12 September 2020}

Published online: 30 September 2020

\section{References}

1. Neal B, Perkovic V, Mahaffey KW, de Zeeuw D, Fulcher G, Erondu N, Shaw W, Law G, Desai M, Matthews DR, et al. Canagliflozin and cardiovascular and renal events in type 2 diabetes. N Engl J Med. 2017;377(7):644-57.

2. Zinman B, Wanner C, Lachin JM, Fitchett D, Bluhmki E, Hantel S, Mattheus M, Devins T, Johansen OE, Woerle HJ, et al. Empagliflozin, cardiovascular outcomes, and mortality in type 2 diabetes. N Engl J Med. 2015;373(22):2117-288.

3. Wiviott SD, Raz I, Bonaca MP, Mosenzon O, Kato ET, Cahn A, Silverman MG, Zelniker TA, Kuder JF, Murphy SA, et al. Dapagliflozin and cardiovascular outcomes in type 2 diabetes. N Engl J Med. 2019;380(4):347-57.

4. Kluger AY, Tecson KM, Lee AY, Lerma EV, Rangaswami J, Lepor NE, Cobble ME, McCullough PA. Class effects of SGLT2 inhibitors on cardiorenal outcomes. Cardiovasc Diabetol. 2019:18(1):99.

5. Yuan Z, DeFalco FJ, Ryan PB, Schuemie MJ, Stang PE, Berlin JA, Desai M, Rosenthal N. Risk of lower extremity amputations in people with type 2 diabetes mellitus treated with sodium-glucose co-transporter-2 inhibitors in the USA: A retrospective cohort study. Diabetes Obes Metab. 2018;20(3):582-9.

6. Udell JA, Yuan Z, Rush T, Sicignano NM, Galitz M, Rosenthal N. Cardiovascular outcomes and risks after initiation of a sodium glucose cotransporter 2 inhibitor: results from the EASEL population-based cohort study (evidence for cardiovascular outcomes with sodium glucose cotransporter 2 inhibitors in the real world). Circulation. 2018;137(14):1450-9.

7. Adimadhyam S, Lee TA, Calip GS, Smith Marsh DE, Layden BT, Schumock GT. Risk of amputations associated with SGLT2 inhibitors compared to DPP-4 inhibitors: A propensity-matched cohort study. Diabetes Obes Metab. 2018;20(12):2792-9.

8. Newman JD, Rockman CB, Kosiborod M, Guo Y, Zhong H, Weintraub HS, Schwartzbard AZ, Adelman MA, Berger JS. Diabetes mellitus is a coronary heart disease risk equivalent for peripheral vascular disease. Am Heart J. 2017;184:114-20.

9. Rhee SY, Kim YS. Peripheral arterial disease in patients with type 2 diabetes mellitus. Diabetes Metab J. 2015;39(4):283-90.

10. Belch J, MacCuish A, Campbell I, Cobbe S, Taylor R, Prescott R, Lee R, Bancroft J, MacEwan S, Shepherd J, et al. The prevention of progression of arterial disease and diabetes (POPADAD) trial: factorial randomised placebo controlled trial of aspirin and antioxidants in patients with diabetes and asymptomatic peripheral arterial disease. BMJ. 2008;337:a1840.

11. Marso SP, Hiatt WR. Peripheral arterial disease in patients with diabetes. J Am Coll Cardiol. 2006;47(5):921-9.

12. Verma $S$, Mazer $C D$, Al-Omran $M$, Inzucchi SE, Fitchett $D$, Hehnke $U$, George JT, Zinman B. Cardiovascular outcomes and safety of empagliflozin in patients with type 2 diabetes mellitus and peripheral artery disease: a subanalysis of EMPA-REG OUTCOME. Circulation. 2018;137(4):405-7.

13. Davies MJ, D'Alessio DA, Fradkin J, Kernan WN, Mathieu C, Mingrone G, Rossing $\mathrm{P}$, Tsapas A, Wexler DJ, Buse JB. Management of hyperglycemia in type 2 diabetes, 2018. A consensus report by the american diabetes association (ada) and the European Association for the Study of Diabetes (EASD). Diabetes Care. 2018;41(12):2669-701.

14. Fei Y, Tsoi MF, Cheung BMY. Cardiovascular outcomes in trials of new antidiabetic drug classes: a network meta-analysis. Cardiovasc Diabetol. 2019;18(1):112.

15. National Health Insurance Administration, Ministry of Health and Welfare, Taiwan. National Health Insurance Annual Statistical Report 2018 [updated May 20, 2020. https://www.nhi.gov.tw/English/Content_List. aspx?n=AB41B66610EAC01A\&topn=616B97F8DF2C3614]

16. Austin PC. The use of propensity score methods with survival or time-toevent outcomes: reporting measures of effect similar to those used in randomized experiments. Stat Med. 2014;33(7):1242-58.

17. McCaffrey DF, Griffin BA, Almirall D, Slaughter ME, Ramchand R, Burgette LF. A tutorial on propensity score estimation for multiple treatments using generalized boosted models. Stat Med. 2013;32(19):3388-414.

18. Austin PC. An introduction to propensity score methods for reducing the effects of confounding in observational studies. Multivariate Behav Res. 2011;46(3):399-424.

19. Austin PC. Balance diagnostics for comparing the distribution of baseline covariates between treatment groups in propensity-score matched samples. Stat Med. 2009;28(25):3083-107.

20. Scirica BM, Bhatt DL, Braunwald E, Steg PG, Davidson J, Hirshberg B, Ohman P, Frederich R, Wiviott SD, Hoffman EB, et al. Saxagliptin and cardiovascular outcomes in patients with type 2 diabetes mellitus. N Engl J Med. 2013;369(14):1317-26.

21. Green JB, Bethel MA, Armstrong PW, Buse JB, Engel SS, Garg J, Josse R, Kaufman KD, Koglin J, Korn S, et al. Effect of sitagliptin on cardiovascular outcomes in type 2 diabetes. N Engl J Med. 2015;373(3):232-42.

22. White WB, Cannon CP, Heller SR, Nissen SE, Bergenstal RM, Bakris GL, Perez AT, Fleck PR, Mehta CR, Kupfer S, et al. Alogliptin after acute coronary syndrome in patients with type 2 diabetes. N Engl J Med. 2013;369(14):1327-35.

23. Zelniker TA, Wiviott SD, Raz I, Im K, Goodrich EL, Bonaca MP, Mosenzon $\mathrm{O}$, Kato ET, Cahn A, Furtado RHM, et al. SGLT2 inhibitors for primary and secondary prevention of cardiovascular and renal outcomes in type 2 diabetes: a systematic review and meta-analysis of cardiovascular outcome trials. Lancet. 2019;393(10166):31-9.

24. Pasternak B, Ueda P, Eliasson B, Svensson AM, Franzen S, Gudbjornsdottir S, Hveem K, Jonasson C, Wintzell V, Melbye M, et al. Use of sodium glucose cotransporter 2 inhibitors and risk of major cardiovascular events and heart failure: Scandinavian register based cohort study. BMJ. 2019;366:14772

25. Patorno E, Goldfine AB, Schneeweiss S, Everett BM, Glynn RJ, Liu J, Kim SC. Cardiovascular outcomes associated with canagliflozin versus other non-gliflozin antidiabetic drugs: population based cohort study. BMJ. 2018;360:k119.

26. Kim YG, Han SJ, Kim DJ, Lee KW, Kim HJ. Association between sodium glucose co-transporter 2 inhibitors and a reduced risk of heart failure in patients with type 2 diabetes mellitus: a real-world nationwide population-based cohort study. Cardiovasc Diabetol. 2018;17(1):91.

27. Patorno E, Pawar A, Franklin JM, Najafzadeh M, Deruaz-Luyet A, Brodovicz KG, Sambevski S, Bessette LG, Santiago Ortiz AJ, Kulldorff M, et al. 
Empagliflozin and the risk of heart failure hospitalization in routine clinical care. Circulation. 2019;139(25):2822-30.

28. McMurray JJV, Solomon SD, Inzucchi SE, Kober L, Kosiborod MN, Martinez FA, Ponikowski P, Sabatine MS, Anand IS, Belohlavek J, et al. Dapagliflozin in patients with heart failure and reduced ejection fraction. N Engl J Med. 2019;381 (21):1995-2008.

29. Packer M. Lessons learned from the DAPA-HF trial concerning the mechanisms of benefit of SGLT2 inhibitors on heart failure events in the context of other large-scale trials nearing completion. Cardiovasc Diabetol. 2019;18(1):129.

30. Jude EB, Oyibo SO, Chalmers N, Boulton AJ. Peripheral arterial disease in diabetic and nondiabetic patients: a comparison of severity and outcome. Diabetes Care. 2001;24(8):1433-7.

31. Chatterjee S, Bandyopadhyay D, Ghosh RK, Majumdar U, Aneja A, Lavie CJ, Deedwania P. SGLT-2 inhibitors and peripheral artery disease: a statistical hoax or reality? Curr Probl Cardiol. 2019:44(7):207-22.

32. Chang HY, Singh S, Mansour O, Baksh S, Alexander GC. Association between sodium-glucose cotransporter 2 inhibitors and lower extremity amputation among patients with type 2 diabetes. JAMA Intern Med. 2018:178(9):1190-8.

33. Heyward J, Mansour O, Olson L, Singh S, Alexander GC. Association between sodium-glucose cotransporter 2 (SGLT2) inhibitors and lower extremity amputation: A systematic review and meta-analysis. PLoS ONE. 2020;15(6):e0234065.

34. Lin B, Koibuchi N, Hasegawa Y, Sueta D, Toyama K, Uekawa K, Ma M, Nakagawa T, Kusaka H, Kim-Mitsuyama S. Glycemic control with empagliflozin, a novel selective SGLT2 inhibitor, ameliorates cardiovascular injury and cognitive dysfunction in obese and type 2 diabetic mice. Cardiovasc Diabetol. 2014;13:148.

35. Gaspari T, Spizzo I, Liu H, Hu Y, Simpson RW, Widdop RE, Dear AE. Dapagliflozin attenuates human vascular endothelial cell activation and induces vasorelaxation: A potential mechanism for inhibition of atherogenesis. Diab Vasc Dis Res. 2018;15(1):64-73.

36. Sugiyama S, Jinnouchi H, Kurinami N, Hieshima K, Yoshida A, Jinnouchi K, Nishimura H, Suzuki T, Miyamoto F, Kajiwara K, et al. The SGLT2 inhibitor dapagliflozin significantly improves the peripheral microvascular endothelial function in patients with uncontrolled type 2 diabetes mellitus. Intern Med. 2018;57(15):2147-56.
37. Fuchigami A, Shigiyama F, Kitazawa T, Okada Y, Ichijo T, Higa M, Hiyoshi T, Inoue I, Iso K, Yoshii $\mathrm{H}$, et al. Efficacy of dapagliflozin versus sitagliptin on cardiometabolic risk factors in Japanese patients with type 2 diabetes: a prospective, randomized study (DIVERSITY-CVR). Cardiovasc Diabetol. 2020;19(1):1.

38. Gerhard-Herman MD, Gornik HL, Barrett C, Barshes NR, Corriere MA, Drachman DE, Fleisher LA, Fowkes FGR, Hamburg NM, Kinlay S, et al. 2016 AHA/ACC guideline on the management of patients with lower extremity peripheral artery disease: executive summary: a report of the american college of cardiology/american heart association task force on clinical practice guidelines. J Am Coll Cardiol. 2017;69(11):1465-508.

39. Shao SC, Chang KC, Hung MJ, Yang NI, Chan YY, Chen HY, Kao Yang YH, Lai EC. Comparative risk evaluation for cardiovascular events associated with dapagliflozin vs. empagliflozin in real-world type 2 diabetes patients: a multi-institutional cohort study. Cardiovasc Diabetol. 2019;18(1):120.

40. Suissa S, Azoulay L. Metformin and the risk of cancer: time-related biases in observational studies. Diabetes Care. 2012;35(12):2665-733.

41. Suissa S. Lower Risk of Death With SGLT2 Inhibitors in observational studies: real or bias? Diabetes Care. 2018;41(1):6-10.

42. Zhang Y, Hu G, Yuan Z, Chen L. Glycosylated hemoglobin in relationship to cardiovascular outcomes and death in patients with type 2 diabetes: a systematic review and meta-analysis. PLoS ONE. 2012;7(8):e42551.

43. Hooi JD, Kester AD, Stoffers HE, Overdijk MM, van Ree JW, Knottnerus JA. Incidence of and risk factors for asymptomatic peripheral arterial occlusive disease: a longitudinal study. Am J Epidemiol. 2001;153(7):666-72.

44. Cacoub PP, Abola MT, Baumgartner I, Bhatt DL, Creager MA, Liau CS, Goto S, Rother J, Steg PG, Hirsch AT, et al. Cardiovascular risk factor control and outcomes in peripheral artery disease patients in the Reduction of Atherothrombosis for Continued Health (REACH) Registry. Atherosclerosis. 2009;204(2):e86-92.

\section{Publisher's Note}

Springer Nature remains neutral with regard to jurisdictional claims in published maps and institutional affiliations.
Ready to submit your research? Choose BMC and benefit from:

- fast, convenient online submission

- thorough peer review by experienced researchers in your field

- rapid publication on acceptance

- support for research data, including large and complex data types

- gold Open Access which fosters wider collaboration and increased citations

- maximum visibility for your research: over $100 \mathrm{M}$ website views per year

At BMC, research is always in progress.

Learn more biomedcentral.com/submissions 